\title{
HD 149277: a rare short-period SB2 system with a subsynchronously rotating magnetic He-rich primary ${ }^{\star}$.
}

\author{
J. F. González, ${ }^{1} \dagger$ S. Hubrig, ${ }^{2} \ddagger$ S. P. Järvinen ${ }^{2}$, M. Schöller ${ }^{3}$ \\ ${ }^{1}$ Instituto de Ciencias Astronómicas, de la Tierra y del Espacio (ICATE), Av. España Sur 1512, CC 49, 5400 San Juan, Argentina \\ ${ }^{2}$ Leibniz-Institut für Astrophysik, An der Sternwarte 16, 14482 Potsdam, Germany \\ ${ }^{3}$ European Southern Observatory, Karl-Schwarzschild-Str. 2, 85748 Garching, Germany
}

Accepted XXX. Received YYY; in original form ZZZ

\begin{abstract}
HD 149277 is a rare SB2 system with a slowly rotating magnetic He-rich primary with $P_{\text {rot }}=25.4 \mathrm{~d}$. The CFHT/ESPaDOnS archive spectra revealed $P_{\text {orb }}=11.5192 \pm 0.0005 \mathrm{~d}$ indicating strong subsynchronous rotation of the primary component. Such a strong subsynchronous rotation was not detected in any other SB2 system with a magnetic chemically peculiar component. Our inspection of the spectra revealed the presence of resolved Zeeman split spectral lines allowing us to determine the variability of the mean magnetic field modulus over the rotation period. The maximum of the magnetic field modulus concides roughly with the positive extremum of the longitudinal field, whereas the minimum of the modulus with the negative extremum of the longitudinal field. No evidence for a longitudinal magnetic field was seen in the circularly polarized spectra of the secondary component. Using archival data from the ASAS3 survey, we find in the frequency spectrum only one significant peak, corresponding to the period $P_{\text {phot }}=$ $25.390 \pm 0.014 \mathrm{~d}$. This value is in good agreements with the previous determination of the rotation period, $P_{\text {rot }}=25.380 \pm 0.007 \mathrm{~d}$, which was based on longitudinal magnetic field measurements.
\end{abstract}

Key words: stars: individual: HD 149277 - stars: magnetic fields - stars: chemically peculiar - stars: rotation - stars: binaries: spectroscopic - stars: massive

\section{INTRODUCTION}

Using high-resolution polarimetric spectra of HD 149277 acquired with ESPaDOnS at the Canada-France-Hawaii Telescope (CFHT), Shultz et al. (2018) recently reported on the presence of a rather strong longitudinal magnetic field with $\left\langle B_{\mathrm{z}}\right\rangle_{\max }=3.3 \pm 0.1 \mathrm{kG}$. The authors determined a rotation period of $25.4 \mathrm{~d}$ using these mean longitudinal magnetic field measurements. HD 149277 is a member of the young open cluster NGC6178 at an age of $\log (t[\mathrm{yr}])=$ $7.15 \pm 0.22$ (Kharchenko et al. 2005). Its physical properties, $T_{\text {eff }}=22300 \pm 500 \mathrm{~K}, M=8.75 \pm 0.40 \mathrm{M}_{\odot}$, and $\log \left(L / L_{\odot}\right)=$ $3.7 \pm 0.1$ were reported by Landstreet et al. (2007). In the column "remarks" of their Table 1, Shultz et al. (2018) mention that HD 149277 is an SB2 system with a B2IV/V mag-

\footnotetext{
* Based on observations obtained at the Canada-France-Hawaii Telescope (CFHT) which is operated by the National Research Council of Canada, the Institut National des Sciences de l'Univers of the Centre National de la Recherche Scientique of France, and the University of Hawaii.

$\dagger$ E-mail: jfgonzalez@conicet.gov.ar

† E-mail: shubrig@aip.de
}

netic primary, but give no information on the nature of the secondary component or the orbital parameters.

According to previous studies by e.g. Carrier et al. (2002), Hubrig et al. (2014), and Landstreet et al. (2017), close SB2 systems with magnetic Ap or Bp components are extremely rare. Among the studied binary systems with A and late B-type primaries, only two systems, HD 98088 and HD 161701, are known to possess a magnetic Ap star as a companion (Babcock 1958; Abt et al. 1968; Hubrig et al. 2014), and only a few more SB2 systems with Bp components are currently known (Landstreet et al. 2017). Such systems are of considerable interest in the context of the magnetic field origin, which is still not properly understood. Ferrario et al. (2009) suggested that magnetic Ap and Bp stars are products of a merger of two lower mass protostars. In this scenario, the binaries that we observe now were triple systems earlier in their history. In the following we report on our analysis of the orbital parameters of HD 149277 and give additional information on the spectral, magnetic, and photometric variability. 
Table 1. Radial velocity measurements carried out for the primary and secondary components in the system HD 149277. The first observation corresponds to the HARPS spectrum.

\begin{tabular}{|c|c|c|c|}
\hline $\begin{array}{c}\text { HJD } \\
2450000+\end{array}$ & $\begin{array}{l}\text { Orbital } \\
\text { Phase }\end{array}$ & $\begin{array}{c}R V_{\mathrm{A}} \\
\left(\mathrm{km} \mathrm{s}^{-1}\right)\end{array}$ & $\begin{array}{c}R V_{\mathrm{B}} \\
\left(\mathrm{km} \mathrm{s}^{-1}\right)\end{array}$ \\
\hline 4206.3982 & 0.636 & $69.3 \pm 0.3$ & $-79.7 \pm 0.5$ \\
\hline 6112.3310 & 0.103 & $-113.0 \pm 0.3$ & $119.4 \pm 0.4$ \\
\hline 6759.6146 & 0.298 & $-75.4 \pm 0.3$ & $76.1 \pm 0.5$ \\
\hline 6760.4658 & 0.372 & $-36.9 \pm 0.3$ & $33.6 \pm 0.6$ \\
\hline 6812.3925 & 0.880 & $71.6 \pm 0.3$ & $-83.0 \pm 0.5$ \\
\hline 6818.3859 & 0.400 & $-21.6 \pm 0.3$ & $18.7 \pm 0.4$ \\
\hline 6821.3857 & 0.660 & $74.7 \pm 0.3$ & $-87.5 \pm 0.5$ \\
\hline 6822.3910 & 0.748 & $88.7 \pm 0.3$ & $-103.2 \pm 0.7$ \\
\hline 6824.3772 & 0.920 & $51.1 \pm 0.3$ & $-61.8 \pm 0.5$ \\
\hline 7225.2883 & 0.726 & $87.0 \pm 0.3$ & $-100.4 \pm 0.5$ \\
\hline 7226.2343 & 0.808 & $88.8 \pm 0.3$ & $-103.7 \pm 0.5$ \\
\hline 7227.2542 & 0.896 & $64.4 \pm 0.3$ & $-77.4 \pm 0.5$ \\
\hline 7228.2936 & 0.987 & $-4.1 \pm 0.4$ & $-1.0 \pm 0.9$ \\
\hline 7229.3121 & 0.075 & $-93.2 \pm 0.3$ & $98.0 \pm 0.5$ \\
\hline 7230.3155 & 0.162 & $-125.4 \pm 0.4$ & $134.4 \pm 0.8$ \\
\hline 7231.2850 & 0.246 & $-100.0 \pm 0.4$ & $104.8 \pm 0.5$ \\
\hline 7232.3179 & 0.336 & $-54.0 \pm 0.4$ & $55.4 \pm 0.5$ \\
\hline 7233.2322 & 0.415 & $-14.0 \pm 0.4$ & $9.1 \pm 0.5$ \\
\hline 7234.2364 & 0.503 & $24.4 \pm 0.4$ & $-32.6 \pm 0.7$ \\
\hline 7235.2543 & 0.591 & $55.8 \pm 0.4$ & $-66.5 \pm 0.5$ \\
\hline 7236.3116 & 0.683 & $79.5 \pm 0.3$ & $-93.3 \pm 0.5$ \\
\hline 7604.2298 & 0.624 & $66.6 \pm 0.3$ & $-78.3 \pm 0.5$ \\
\hline 7611.2236 & 0.231 & $-106.5 \pm 0.4$ & $112.6 \pm 0.5$ \\
\hline 7611.2503 & 0.234 & $-105.4 \pm 0.4$ & $111.4 \pm 0.5$ \\
\hline
\end{tabular}

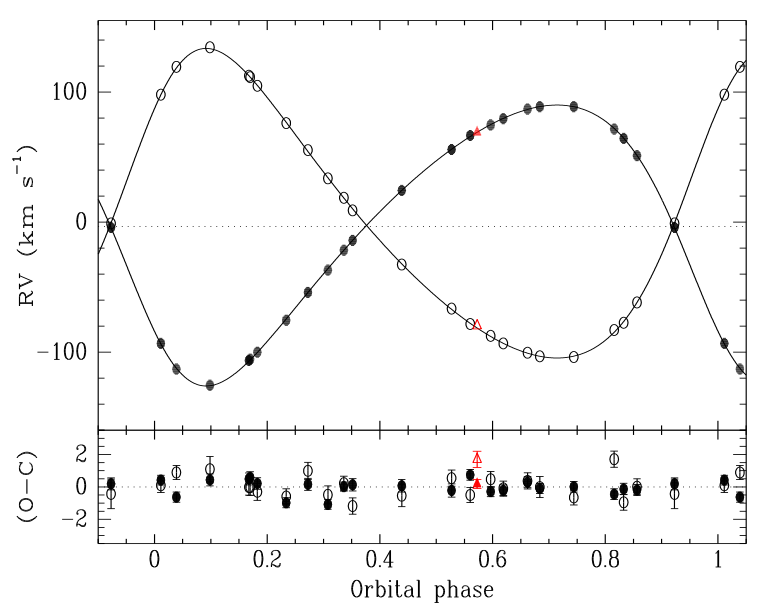

Figure 1. Spectroscopic orbit of the system HD 149277 using ESPaDOnS spectra obtained from 2012 to 2016 and one HARPS spectrum (red triangles). Open symbols indicate measurements of the secondary component, which is the less massive star. The lower panel shows the residuals: observed minus calculated. Phase zero corresponds to the periastron passage. In the upper panel error bars are smaller than the symbol sizes.

\section{ORBITAL ANALYSIS}

To estimate the orbital parameters of the SB2 system HD 149277, we downloaded all publically available ESPaDOnS (Echelle SpectroPolarimetric Device for the Observation of Stars) spectra obtained from 2012 to 2016. ES$\mathrm{PaDOnS}$ is a spectropolarimeter with a resolving power of
Table 2. Orbital and fundamental parameters for the SB2 system HD 149277.

\begin{tabular}{cc}
\hline$P(\mathrm{~d})$ & $11.5192 \pm 0.0005$ \\
$T_{\text {conj }}$ & $2456802.77 \pm 0.02$ \\
$T_{\mathrm{per}}$ & $2456803.51 \pm 0.06$ \\
$V_{\mathrm{o}}\left(\mathrm{km} \mathrm{s}^{-1}\right)$ & $-2.6 \pm 0.3$ \\
$K_{A}\left(\mathrm{~km} \mathrm{~s}^{-1}\right)$ & $107.8 \pm 0.7$ \\
$K_{B}\left(\mathrm{~km} \mathrm{~s}^{-1}\right)$ & $118.2 \pm 0.8$ \\
$\omega$ & $2.23 \pm 0.03$ \\
$e$ & $0.236 \pm 0.004$ \\
$a \sin i\left(R_{\odot}\right)$ & $50.0 \pm 0.2$ \\
$M \sin ^{3} i\left(M_{\odot}\right)$ & $12.66 \pm 0.16$ \\
$M_{\mathrm{A}} \sin ^{3} i\left(M_{\odot}\right)$ & $6.62 \pm 0.09$ \\
$M_{\mathrm{B}} \sin ^{3} i\left(M_{\odot}\right)$ & $6.04 \pm 0.08$ \\
$q$ & $0.912 \pm 0.007$ \\
\hline$v \sin i_{\mathrm{A}}\left(\mathrm{km} \mathrm{s}^{-1}\right)$ & $8 \pm 3^{a}$ \\
$v \sin i_{\mathrm{B}}\left(\mathrm{km} \mathrm{s}^{-1}\right)$ & $11.5 \pm 4$ \\
\hline
\end{tabular}

${ }^{a}$ Shultz et al. (2018).

about 65000 , covering the wavelength region from 3700 to $10500 \AA$. As most of the spectra have a rather low signal-tonoise ratio $(\mathrm{S} / \mathrm{N})$ between 85 and 100 , we averaged spectra recorded during the same night (separated by up to $3 \mathrm{~h}$ ) in order to improve the $\mathrm{S} / \mathrm{N}$, obtaining a total of 23 spectra. To this sample we added one available HARPS (High Accuracy Radial velocity Planet Searcher) spectrum taken in 2007. In Table 1 we present our radial velocity measurements for the primary and secondary components on 24 phases and in Fig. 1 the spectroscopic orbit of the system. The value $v \sin i_{\mathrm{B}}$ consigned in Table 1 was determined using the $\mathrm{S}$ II 5665 line with a low Landé factor of 0.5 .

Assuming the photometric mass derived by Landstreet et al. (2007), which is consistent with the spectral type, the orbital inclination is estimated to be around $66^{\circ}$.

The derived orbital parameters (see Table 2) reveal that the orbit of HD 149277 is eccentric and that the rotation of the components is not synchronized. In the case of pseudosynchronization we would expect $P_{\text {pseudosync }}=8.92 \mathrm{~d}$. The primary component shows strong subsynchronous rotation, most probably caused by magnetic breaking in a system without strong rotation-orbital coupling. Given the mass ratio of 0.912 , both components are early B-type stars. The secondary is probably by $1000 \mathrm{~K}$ cooler with a spectral type $\mathrm{B} 3^{-} \mathrm{V}$.

\section{MAGNETIC FIELD MEASUREMENTS}

One of the most interesting aspects of the system HD 149277 is the rather strong magnetic field and slow rotation of its primary component. Our inspection of the ESPaDOnS spectra revealed the presence of several resolved Zeeman split lines. Their presence has not been reported previously. A number of Ap and late-type Bp stars with strong magnetic fields show resolved Zeeman split spectral lines, allowing to set additional constraints on the magnetic field geometry by measuring the mean magnetic field modulus, i.e. the average over the visible stellar hemisphere of the modulus of the magnetic field vector, weighted by the local line intensity, following the relation given e.g. by Hubrig \& Nesvacil 


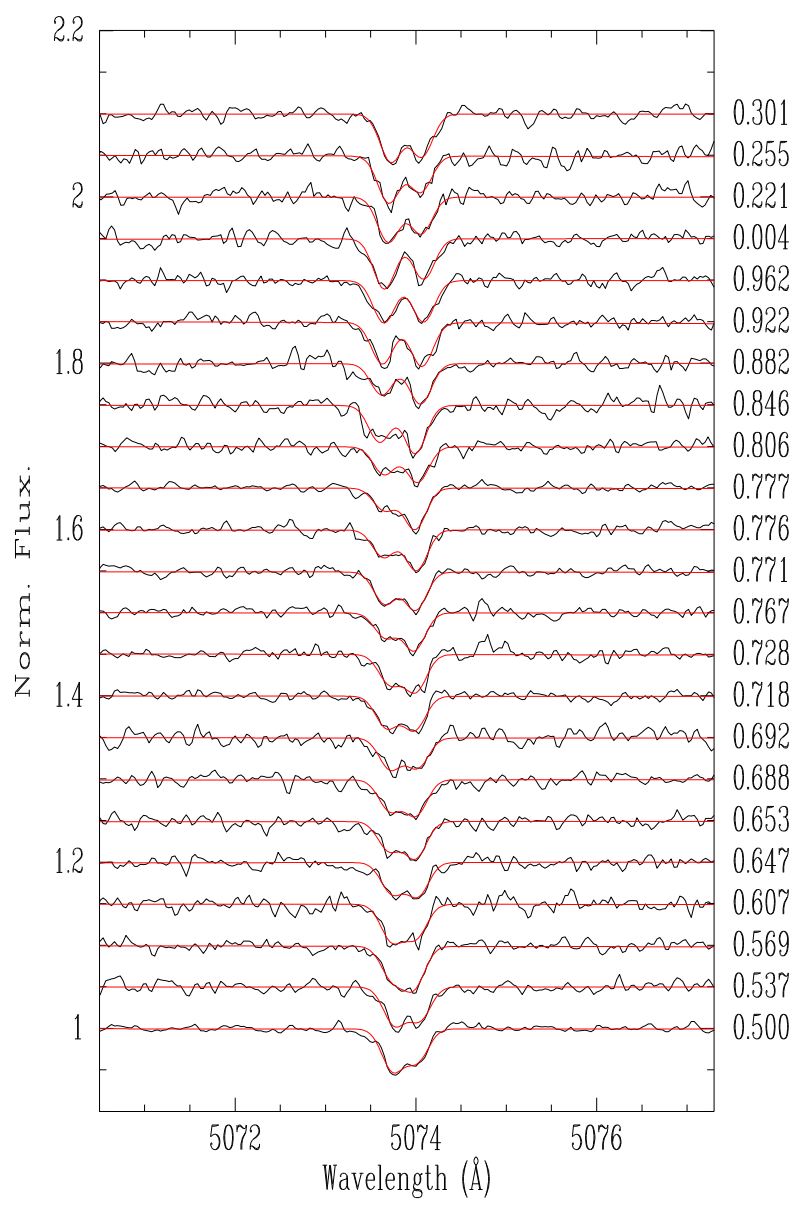

Figure 2. Variability of the magnetically split Fe III 5073.6 line over the rotation period in the disentangled spectra of the primary component. Spectra are vertically offset for better visibility and are sorted in rotational phase (see right side; starting at 0.5 ) from bottom to top. The red lines represent a double-Gaussian fit to the data.

(2007) and Mathys (2017). Such Zeeman split lines can only be seen in stars whose projected rotational velocity is sufficiently small and whose magnetic field is strong enough to exceed the rotational Doppler broadening (Mathys et al. 1997). In Fig. 2 we present the rotational variability of the resolved Zeeman split Fe III 5073.6 line in the primary component. Interestingly, as illustrated in this figure and below in Fig. 4, we observe that in certain phases the Zeeman split lines are asymmetric about the line centers with the blue components appearing less deep than the red components, and with the red components deeper in other phases. Such asymmetries occur in the same way in other transitions, indicating that the rotational Doppler effect is non-negligible, i.e. various parts of the stellar surface characterized by different magnetic field strengths contribute to the line profile.

In order to estimate the mean magnetic field modulus, we fitted the line $\lambda 5073.9$ with two Gaussian profiles. The position and height of both Zeeman components were considered as free parameters, but the Gaussian width was assumed to be the same for both components in all spectra. The use of a fixed value of Gaussian width gives better results in the spectra with lower $\mathrm{S} / \mathrm{N}$ and eases to measure the position of the components even if they are blended. The
Table 3. Measurements of the mean magnetic field modulus in the primary component of HD 149277.

\begin{tabular}{ccc}
\hline $\begin{array}{c}\text { HJD } \\
2450000+\end{array}$ & $\begin{array}{c}\text { Rotational } \\
\text { Phase }\end{array}$ & $\begin{array}{c}\langle B\rangle \\
(\mathrm{kG})\end{array}$ \\
\hline 6112.3310 & 0.718 & $6.32 \pm 0.24$ \\
6759.6146 & 0.221 & $7.48 \pm 0.28$ \\
6760.4658 & 0.255 & $7.27 \pm 0.30$ \\
6812.3925 & 0.301 & $7.01 \pm 0.24$ \\
6818.3859 & 0.537 & $5.79 \pm 0.34$ \\
6821.3857 & 0.655 & $6.08 \pm 0.36$ \\
6822.3910 & 0.695 & $6.23 \pm 0.49$ \\
6824.3772 & 0.773 & $6.96 \pm 0.22$ \\
7225.2883 & 0.569 & $5.00 \pm 0.37$ \\
7226.2343 & 0.607 & $5.51 \pm 0.39$ \\
7227.2542 & 0.647 & $5.90 \pm 0.37$ \\
7228.2936 & 0.688 & $6.01 \pm 0.41$ \\
7229.3121 & 0.728 & $5.92 \pm 0.36$ \\
7230.3155 & 0.767 & $6.55 \pm 0.36$ \\
7231.2850 & 0.806 & $7.60 \pm 0.32$ \\
7232.3179 & 0.846 & $8.11 \pm 0.34$ \\
7233.2322 & 0.882 & $8.80 \pm 0.31$ \\
7234.2364 & 0.922 & $9.03 \pm 0.24$ \\
7235.2543 & 0.962 & $9.04 \pm 0.22$ \\
7236.3117 & 0.004 & $8.94 \pm 0.22$ \\
7604.2298 & 0.500 & $5.64 \pm 0.23$ \\
7611.2236 & 0.776 & $7.46 \pm 0.26$ \\
7611.2503 & 0.777 & $6.96 \pm 0.28$ \\
\hline
\end{tabular}

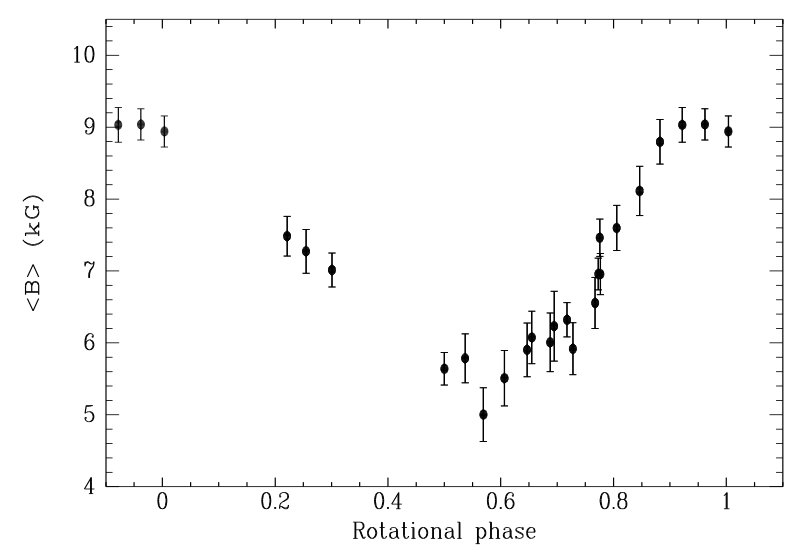

Figure 3. Mean magnetic field modulus, calculated from the Zeeman split Fe III 5073.6 line, over the rotation of the primary component of HD 149277.

values of the mean magnetic field modulus (see Table 3 and Fig. 3) were calculated adopting a Landé factor $g_{\text {eff }}=2.01$, taken from the VALD database (Kupka et al. 2011), and are comprised between 5.0 and $9.0 \mathrm{kG}$. These measurements were cross-checked with measurements of the Zeeman split FeII 5018.4 line, which however affected by low $\mathrm{S} / \mathrm{N}$ and blending with the spectral lines of the secondary in several rotation phases.

The maximum of the mean magnetic field modulus coincides roughly with the positive extremum of the longitudinal magnetic field, whereas the minimum of the modulus coincides with the negative extremum of the longitudinal magnetic field. The phase curve for the longitudinal magnetic field is presented in Fig. A20 in the work of Shultz et al. 


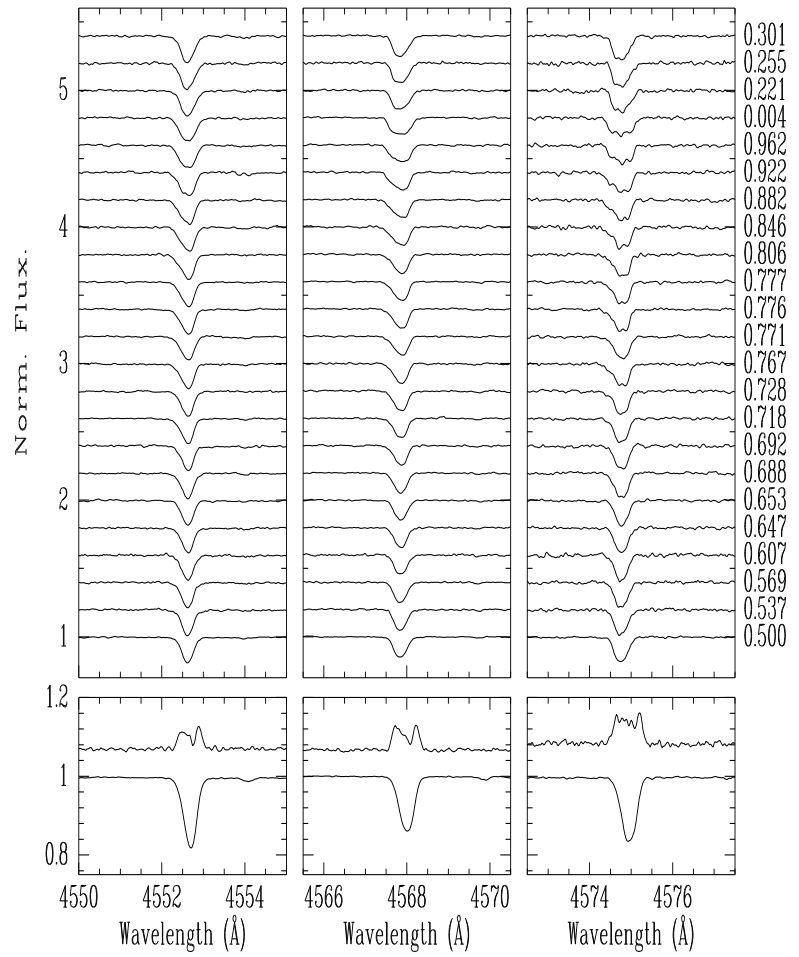

Figure 4. Variability of three Si III line profiles in the disentangled spectra of the primary component of HD 149277. Labels on the right refer to the rotational phases calculated with $P_{\text {rot }}$ and $J D 0$ given by Shultz et al. (2018). The spectra have been shifted vertically for better visibility. The spectra of the faintest line $\lambda 4575$ have been scaled by a factor 2 . The lower panels show the mean profile and the RMS of residuals (scaled $\times 5$ )

(2018). Future spectroscopic monitoring at higher S/N will be worthwhile to better characterize the variability of the mean magnetic field modulus in this component. No evidence for a longitudinal magnetic field was seen in the circularly polarized spectra of the secondary component.

\section{SPECTRAL AND PHOTOMETRIC VARIABILITY}

Like other magnetic Bp stars, HD 149277 exhibits distinct changes in the line profiles of different elements, probably caused by an inhomogeneous element distribution on the stellar surface. As an example, nitrogen lines become stronger at the phases conciding with the negative extremum of the longitudinal magnetic field. The clearest variations are observed in the profiles of the Si III lines. The behaviour of three Si III line profiles in the disentangled spectra of the primary is presented in Fig. 4.

Since spectral lines of the secondary component are weaker and in many cases are overlapped with variable lines of the primary, the existence of spectral variability cannot definitely be established. In Fig. 5 we present the profiles of the Si III 4553 line recorded in the spectra of the secondary component on different orbital phases. Missing parts in the spectra correspond to the position of spectral lines of the primary star (all lines deeper than $1 \%$ of the continuum
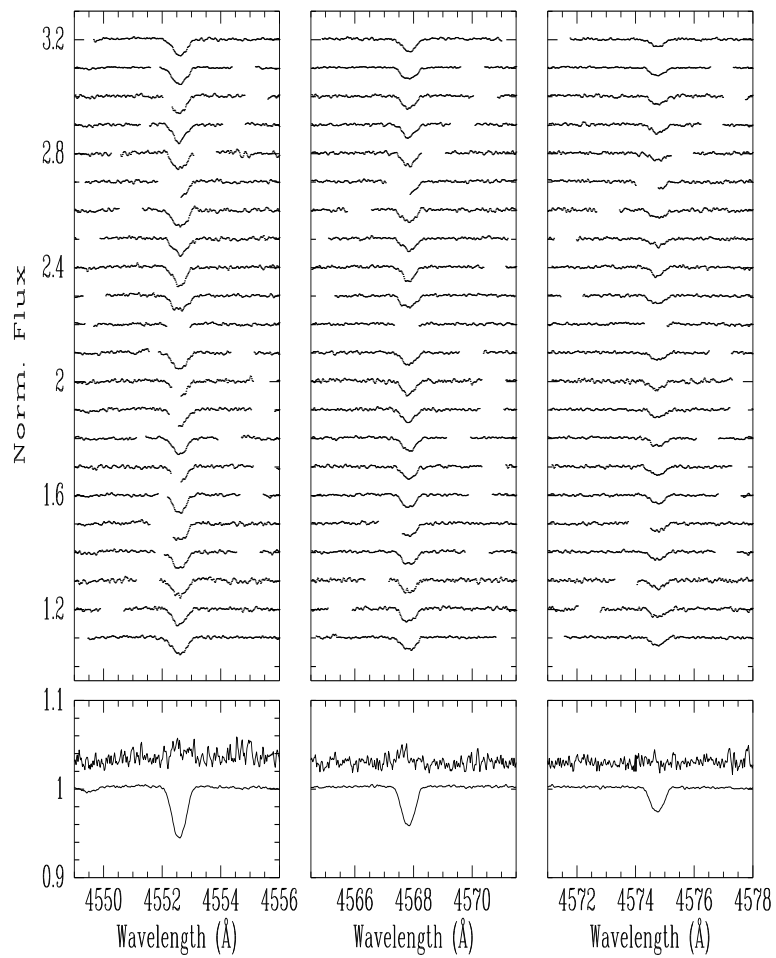

Figure 5. Line profile of Si III 4553 in the disentangled spectra of the secondary component of HD 149277. The spectra are ordered by JD from bottom to top. The spectra of the faintest line $\lambda 4575$ have been scaled by a factor 2 . The lower panels show the mean profile and the RMS of residuals (scaled $\times 10$ )

level). Since lines of the primary are certainly variable these regions were not considered in the calculation of the RMS (lower panel). The rotation period of this component could probably be determined from observed line profile variations, provided higher $\mathrm{S} / \mathrm{N}$ spectra of this system are recorded in future observations. An analysis of the spectral variability of the secondary is of special interest: for the majority of the previously studied close binary systems with $P_{\text {orb }} \leq 20$ d possessing one magnetic or chemically peculiar spectrum variable component, the other component is usually an Am star or a normal A or B-type star. To our knowledge, there are only three exceptions: the system 41 Eri consisting of two spectrum variable HgMn stars (Hubrig et al. 2012), the system HD 161701 consisting of a spectrum variable HgMn star and a magnetic Ap star (Hubrig et al. 2014), and the system HD 136504 consisting of two magnetic Bp stars. The magnetic field in the primary of HD 136504 was detected in low-resolution FORS 2 observations by Hubrig et al. (2010), while the discovery of a magnetic field in the secondary was announced by Shultz et al. (2015).

He-rich stars and chemically peculiar stars usually exhibit photometric variability due to the presence of surface chemical abundance spots. Therefore, rotation periods can also be determined from photometry. Using archival data from the ASAS3 survey ${ }^{1}$ (Pojmanski 1997), we find in the frequency spectrum only one significant peak, $P_{\text {phot }}=$

\footnotetext{
1 http://www.astrouw.edu.pl/asas/
} 

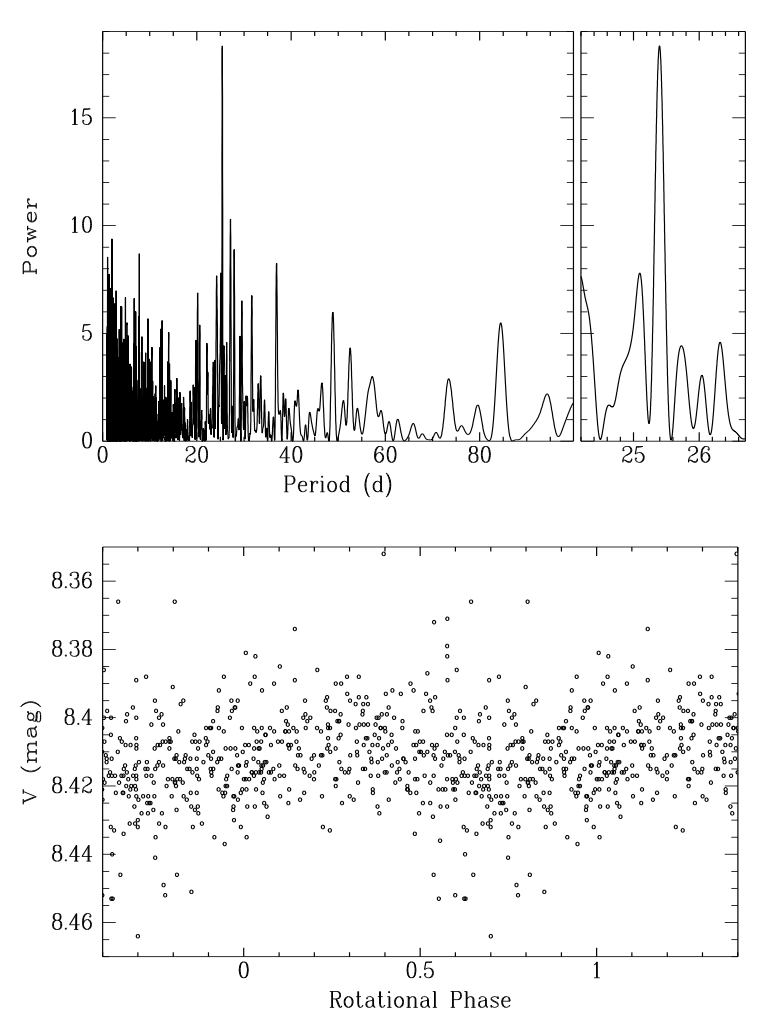

Figure 6. Photometric variability of the primary. Upper panel: Periodogram obtained using the ASAS3 database. Lower panel: The ASAS3 light curve using $P_{\text {phot }}=25.390 \mathrm{~d}$.

$25.390 \pm 0.014 \mathrm{~d}$ (see Fig. 6). This value is in good agreements with the previous determination of the rotation period, $P_{\text {rot }}=25.380 \pm 0.007 \mathrm{~d}$ by Shultz et al. (2018), which was based on longitudinal magnetic field measurements. The photometric variability of this object with a light curve amplitude of $0.010 \pm 0.005 \mathrm{mag}$ is reported here for the first time.

\section{DISCUSSION}

The analysis of the system HD 149277 is of considerable interest as close main sequence SB2 systems only very rarely contain a magnetic Ap or Bp star as a component. We determined the orbital parameters of the system and studied a few characteristics of the individual components, such as spectroscopic and photometric variability. Similar to a few other SB2 systems with magnetic Bp components compiled in Table 5 in the work of Landstreet et al. (2017), the orbit is quite eccentric with $e=0.237$, but the rotation period of the primary is the longest among the studied systems. The more massive of the two stars is rotating subsynchronously and exhibits a strongly variable magnetic field modulus. Among the previously studied SB2 systems, the primary in HD 149277 exhibits the strongest magnetic field and the strongest subsynchronous rotation. The less massive component is apparently non-magnetic, but probably shows spectral variability, which should be analysed in more detail in future higher $\mathrm{S} / \mathrm{N}$ observations. Such future high S/N observations should also be used to constrain the structure of the magnetic field of the primary in more detail.
We note that studies of strongly magnetic stars using the mean magnetic field modulus, measured using magnetically resolved lines, are of importance because they provide the best opportunity to study the effect of a magnetic field on stellar atmospheres. At present, 84 Ap stars are known to show magnetically resolved lines, representing $2.3 \%$ of the total number of known Ap stars (Mathys 2017). The sample of early-type massive Bp stars currently consists of just three members (Hubrig et al., in preparation).

\section{ACKNOWLEDGEMENTS}

This work has made use of the VALD database, operated at Uppsala University, the Institute of Astronomy RAS in Moscow, and the University of Vienna. Based on observations made with ESO Telescopes at the La Silla Paranal Observatory under programme ID 079.D-0118(A). JFG thanks financial support from CONICET (PIP 0331) and the Universidad Nacional de San Juan (Proyecto CICITCA), Argentina

\section{REFERENCES}

Abt H. A., Conti P. S., Deutsch A. J., Wallerstein G., 1968, ApJ, 153, 177

Babcock H. W., 1958, ApJS, 3, 141

Carrier F., North P., Udry S., Babel J., 2002, A\&A, 394, 151

Ferrario L., Pringle J. E., Tout C. A., Wickramasinghe D. T., 2009, MNRAS, 400, L71

Hubrig S., Nesvacil N., 2007, MNRAS, 378, L16

Hubrig S., Ilyin I., Schöller M., Briquet M., Morel T., De Cat P., 2010, ApJ, 726, L5

Hubrig S., et al., 2012, A\&A, 547, A90

Hubrig S., et al., 2014, MNRAS, 440, L6

Kharchenko N. V., Piskunov A. E., Röser S., Schilbach E., Scholz R.-D., 2005, A\&A, 438, 1163

Kupka F., Dubernet M.-L., VAMDC Collaboration, 2011, Baltic Astronomy, 20, 503

Landstreet J. D., Bagnulo S., Andretta V., Fossati L., Mason E., Silaj J., Wade G. A., 2007, A\&A, 470, 685

Landstreet J. D., Kochukhov O., Alecian E., Bailey J. D., Mathis S., Neiner C., Wade G. A., BINaMIcS Collaboration, 2017, A\&A, 601, A129

Mathys G., Hubrig S., Landstreet J. D., Lanz T., Manfroid J., 1997, A\&AS, 123, 353

Mathys G., 2017, A\&A, 601, A14

Pojmanski G., 1997, AcA, 47, 467

Shultz M., Wade G. A., Alecian E., BinaMIcS Collaboration, 2015, MNRAS, 454, L1

Shultz M., et al., 2018, MNRAS, 475, 5144

This paper has been typeset from a TEX/LATEX file prepared by the author. 